\title{
Computation of ion production rate induced by cosmic rays during Bastille day ground level enhancement
}

\author{
Alexander Mishev ${ }^{* \dagger}$ \\ ReSolve CoE University of Oulu, Finland. E-mail: alexander.mishev@oulu.fi \\ Peter I.Y.I Velinov \\ Institute for Space Research and Technology, Bulgarian Academy of Sciences. E-mail: \\ pvelinov@bas.bg
}

The galactic cosmic rays are the main source of ionization in the Earth stratosphere and troposphere. They play an important role in various processes related to atmospheric physics and chemistry. Sporadically solar energetic particles enhance the ion production, specifically over polar caps. At recent was observed an apparent effect on minor constituents and aerosols over polar region during major solar proton events, specifically during the major event of 20 January 2005. Solar cycle 23 provided several strong ground level enhancements. The Bastille day event on 14 July 2000 is among the strongest events of the cycle. In the work presented here we apply a full Monte Carlo 3-D model for cosmic ray induced ionization in order to compute the ion production during the Bastille day event. The model is based on atmospheric cascade simulation with CORSIKA code using FLUKA and QGSJET II hadron generators. The ion production rate during the event is considered as a superposition of cosmic rays with galactic and solar origin. The time evolution of ion production is computed considering the variation of solar proton spectra throughout the event, apparent source position and anisotropy. The ion production rate is computed as a function of the altitude above the sea level at several rigidity cut-offs, namely $1 \mathrm{GV}, 2 \mathrm{GV}$ and 3 $\mathrm{GV}$. The ionization effect is also estimated.

The 34th International Cosmic Ray Conference,

30 July- 6 August, 2015

The Hague, The Netherlands

\footnotetext{
* Speaker.

$\dagger$ also at Institute for Nuclear Research and Nuclear Energy, Bulgarian Academy of Sciences.
} 


\section{Introduction}

The main source of ionization in the troposphere and stratosphere of the Earth are the galactic cosmic rays (GCRS) [1, 2, 3]. They enter in the atmosphere and produce large amount of secondaries. Their intensity is modulated by the solar wind. It follows the 11-year solar cycle and

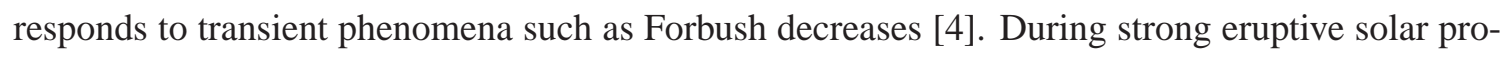
cesses as solar flares and coronal mass ejections (CMEs) are produced solar energetic particles (SEPs) (see [5, 6] and references therein), which is eventually are accelerated to energies enough to initiate an atmospheric cascade, known as ground level enhancements (GLEs). They significantly increase the ion production in the atmosphere, specifically in polar regions [1, 8, 0, 10, 11].

\section{Model, ion production rate and ionization effect during GLE 59}

The computations in this work are based on a model similar to [12]. The detailed are given elsewhere [13, 14, 15].

$$
Q\left(h, \lambda_{m}\right)=\frac{1}{E_{\text {ion }}} \sum_{i} \int_{E}^{\infty} \int_{\Omega} D_{i}(E) \frac{\triangle E(h, E)}{\triangle h} \rho(h) d E d \Omega
$$

where $\triangle E$ is the deposited energy in an atmospheric layer $\triangle h, h$ is the air overburden (air mass) above a given altitude in the atmosphere expressed in $\mathrm{g} / \mathrm{cm}^{2}$ subsequently converted to altitude a.s.l., $D_{i}(E)$ is the differential cosmic ray spectrum for a given nuclei of primary CR, $\rho$ is the atmospheric density in ${\mathrm{g} . \mathrm{cm}^{-3}}^{-3} \lambda_{m}$ is the geomagnetic latitude, $E$ is the initial energy of the incoming primary nuclei on the top of the atmosphere, $\Omega$ is the geometry factor - a solid angle and $E_{\text {ion }}=35$ $\mathrm{eV}$ is the energy necessary for creation of an ion pair in air.

July 2000 was period of intense solar activity, producing three X-class flares and two halo CMEs. The GLE 59 was related to the Bastille day X5.8/3B solar ïńCare and associated full halo CME, started at 10:03 UT, reached peak at 10:24 UT and ended at 10:43 UT [16]. The GLE onset began between 10:30 and 10:35 UT at several stations. The strongest NM increases were observed at South Pole $58.3 \%$ and SANAE $54.4 \%$. In this study we consider SEPs spectra and anisotropy according to reconstructions from neutron monitor data [17]. The ion production rate during the GLE 59 is a superpose of the contribution of GCRs and SEPs. For the GCR spectrum we assume the force field approximation [18, 19, 20]. The computations are fulfilled at realistic conditions, namely assuming a summer atmospheric profile [21, 22, 23]. The computed ion production rate is presented in Fig.1a for $R_{c} \leq 1 \mathrm{GV}$ cut-off, accordingly Fig. $1 \mathrm{~b}$ for $R_{c} \leq 2 \mathrm{GV}$.

The derived ion production rate allow us to estimate the expected ionization effect by integration of ion production rate over the event [24]. The ionization effect is presented in Fig.2 for regions with $R_{c} \leq 1 \mathrm{GV}, R_{c} \leq 2 \mathrm{GV}$ and $R_{c} \leq 3 \mathrm{GV}$ rigidity cut-offs.

It was recently shown that the ion production and the corresponding ionization effect during major GLEs considerably vary throughout the event [25, 26]. This is due on variation of spectral (SEP spectrum soften during the event) and angular characteristics (the pitch angle distribution broaden out). The anisotropy of SEPs considerably reflects on the magnitude of ionization effect in a given geographic region. Since the Bastille day event isotropizes relatively fast, this effect is 


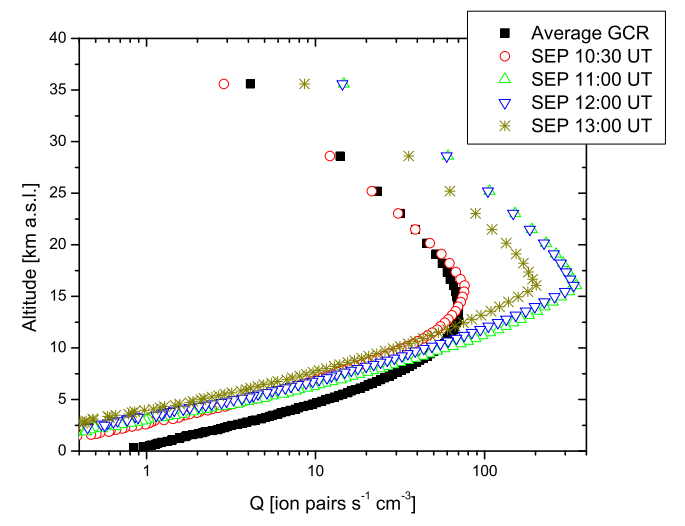

(a) Ion production at $R_{c} \leq 1 \mathrm{GV}$

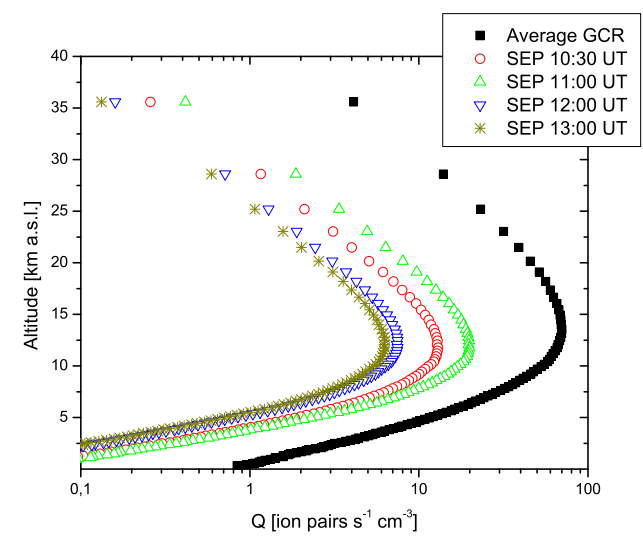

(b) Ion production at $R_{C} \leq 2 \mathrm{GV}$

Figure 1: Ion production rate during the Bastille day GLE on 14 July 2000.

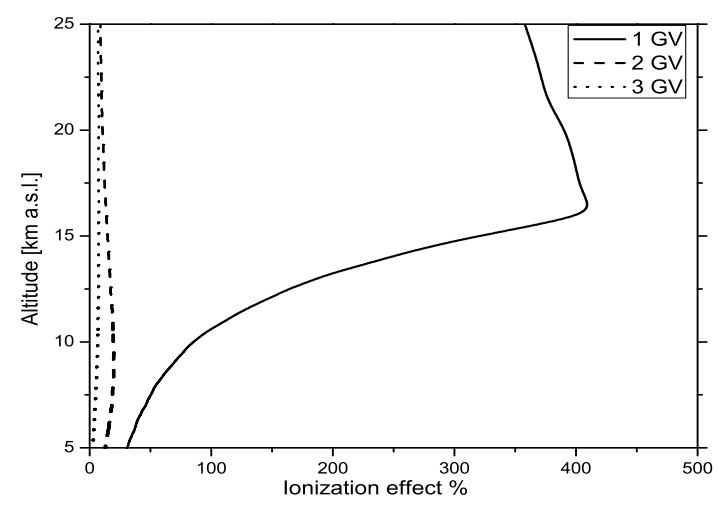

Figure 2: Maximal ionization effect during the Bastille day GLE on 14 July 2000 at $R_{c} \leq 1 \mathrm{GV}, R_{c}$ $\leq 2 \mathrm{GV}$ and $R_{c} \leq 3 \mathrm{GV}$

not as important compared to other events [7, 25]. The ion production is maximal in the sub-polar and polar regions of Southern hemisphere, while it is minimal near to the anti-sunward direction.

As example we compute the ionization effect in the troposphere at altitude of about $10 \mathrm{~km}$ above the sea level throughout the event (Fig.3). The maximal effect ionization effect at this altitude is observed is $\approx 20 \%$ in the region of $30^{\circ} \mathrm{W}-30^{\circ} \mathrm{E}$ in the Northern hemisphere. It diminish to lees then $10 \%$ in anti-sunward direction in the Southern hemisphere.

\section{Conclusion}

In this study we presented computation of ion production rate and corresponding ionization effect due to CRs of galactic and solar origin during the Bastille day GLE event on 14 July 2000. It was shown that the ion production is maximal in the sub-polar and polar regions. It rapidly 


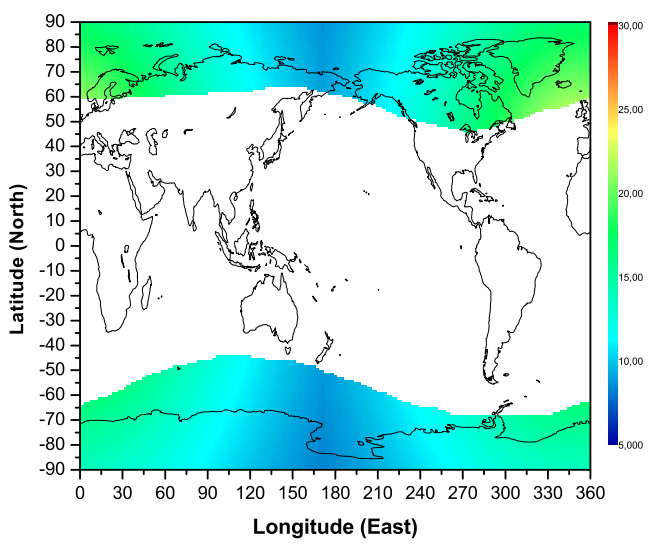

Figure 3: Ionization effect in the troposphere relative to average due to GCR during GLE 59.

diminish at regions with higher rigidity cut-offs, because of the falling SEP spectra. The estimated ionization effect is important for recent studies related to impact of CRs on atmospheric chemistry and physics as well as space weather and space climate [10, 27, 28].

\section{Acknowledgements}

This work was supported by the Center of Excellence ReSoLVE (project No. 272157).

\section{References}

[1] G. A. Bazilevskaya, I. G. Usoskin, E. Flückiger, R. Harrison, L. Desorgher, B. Bütikofer, M. Krainev, V. Makhmutov, Y. Stozhkov, A. Svirzhevskaya, N. Svirzhevsky, and G. Kovaltsov, Cosmic ray induced ion production in the atmosphere, Space Science Reviews 137 (2008) 149-173.

[2] I. G. Usoskin, L. Desorgher, P. Velinov, M. Storini, E. Flückiger, R. Bütikofer, and G. Kovaltsov, Ionization of the Earth's atmosphere by solar and galactic cosmic rays, Acta Geophysica 57 (2009), no. $188-101$.

[3] P. Velinov, S. Asenovski, K. Kudela, J. Lastovička, L. Mateev, A. Mishev, and P. Tonev, Impact of cosmic rays and solar energetic particles on the earth's ionosphere and atmosphere, Journal of Space Weather and Space Climate 3 (2013).

[4] S. Forbush, On the effects in cosmic-ray intensity observed during the recent magnetic storm, Physical Review 51 (1937), no. 121108.

[5] M. Aschwanden, GeV particle acceleration in solar flares and ground level enhancement (gle) events, Space Science Reviews 171 (2012), no. 1-4 3-21.

[6] L. Miroshnichenko, Solar Cosmic Rays: Fundamentals and Applications. Springer, Dordrecht, 2014.

[7] A. Mishev, P. Velinov, L. Mateev, and Y. Tassev, Ionization effect of solar protons in the earth atmosphere - case study of the 20 January 2005 SEP event, Advances of Space Research 48 (2011), no. 7 1232-1237. 
[8] I. G. Usoskin, G. Kovaltsov, I. Mironova, A. Tylka, and W. Dietrich, Ionization effect of solar particle gle events in low and middle atmosphere, Atmospheric Chemistry and Physics 11 (2011) 1979-1988.

[9] A. Mishev, P. Velinov, L. Mateev, and Y. Tassev, Ionization effect of nuclei with solar and galactic origin in the earth atmosphere during GLE 69 on 20 January 2005, Journal of Atmospheric and Solar-Terrestrial Physics 89 (2013), no. 1 1-7.

[10] I. Mironova, I. Usoskin, G. Kovaltsov, and S. Petelina, Possible effect of extreme solar energetic particle event of 20 January 2005 on polar stratospheric aerosols: Direct observational evidence, Atmospheric Chemistry and Physics 12 (2012), no. 2 769-778.

[11] P. Velinov and A. Mishev, Comparison of ionization effect in the atmosphere of the earth due to GLE 65 and GLE 69, Journal of Physics: Conference Series 409 (2013), no. 1.

[12] I. Usoskin and G. Kovaltsov, Cosmic ray induced ionization in the atmosphere: Full modeling and practical applications, Journal of Geophysical Research 111 (2006), no. D21206.

[13] P. Velinov, A. Mishev, and L. Mateev, Model for induced ionization by galactic cosmic rays in the earth atmosphere and ionosphere, Advances in Space Research 44 (2009), no. 9 1002-1007.

[14] A. Mishev and P. I. Velinov, Normalized ionization yield function for various nuclei obtained with full Monte Carlo simulations, Advances of Space Research 48 (2011), no. 1 19-24.

[15] A. Mishev and P. Velinov, Influence of hadron and atmospheric models on computation of cosmic ray ionization in the atmosphere-extension to heavy nuclei, Journal of Atmospheric and Solar-Terrestrial Physics 120 (2014) 111-120.

[16] K.-L. Klein, G. Trottet, P. Lantos, and J.-P. DelaboudiniÃ̂́re, Coronal electron acceleration and relativistic proton production during the 14 July 2000 flare and cme, Astronomy and Astrophysics 373 (2001), no. 3 1073-1082.

[17] D. Bombardieri, M. Duldig, K. Michael, and J. Humble, Relativistic proton production during the 2000 july 14 solar event: The case for multiple source mechanisms, Astrophysical Journal 644 (2006), no. 1 565-574.

[18] L. Gleeson and W. Axford, Solar modulation of galactic cosmic rays, Astrophysical Journal 154 (1968) 1011-1026.

[19] R. Caballero-Lopez and H. Moraal, Limitations of the force field equation to describe cosmic ray modulation, Journal of Geophysical Research 109 (2004) A01101.

[20] I. Usoskin, K. Alanko-Huotari, G. Kovaltsov, and K. Mursula, Heliospheric modulation of cosmic rays: Monthly reconstruction for 1951-2004,, Journal of Geophysical Research 110 (2005), no. A12108.

[21] A. Mishev and P. Velinov, Effects of atmospheric profile variations on yield ionization function y in the atmosphere, Comptes Rendus de l'Academie Bulgare des Sciences 61 (2008), no. 5 639-644.

[22] A. Mishev, P. Velinov, V. Yanke, and E. Eroshenko, Effects of different atmospheric profiles on ionization in the earth atmosphere, in Proc. of 31th ICRC Lodz, Poland, 7 -15 July 2009, 2009.

[23] A. Mishev and P. Velinov, The effect of model assumptions on computations of cosmic ray induced ionization in the atmosphere, Journal of Atmospheric and Solar-Terrestrial Physics 72 (2010), no. 5-6 476-481.

[24] A. Mishev and P. Velinov, Ionization rate profiles due to solar and galactic cosmic rays during GLE 59 on Bastille day 14 July 2000, Comptes Rendus de l'Academie Bulgare des Sciences 68 (2015), no. 3 359-366. 
[25] A. Mishev and P. Velinov, A maverick GLE 70 in solar minimum. calculations of enhanced ionization in the atmosphere due to relativistic solar energetic particles, Comptes Rendus de l'Academie Bulgare des Sciences 66 (2013), no. 10 1457-1462.

[26] A. Mishev and P. Velinov, Time evolution of ionization effect due to cosmic rays in terrestrial atmosphere during gle 70, Journal of Atmospheric and Solar-Terrestrial Physics 129 (2015) 78-86.

[27] A. Mishev and J. Stamenov, Present status and further possibilities for space weather studies at BEO Moussala, Journal of Atmospheric and Solar-Terrestrial Physics 70 (2008), no. 2-4 680-685.

[28] A. Mishev, A study of atmospheric processes based on neutron monitor data and cherenkov counter measurements at high mountain altitude, Journal of Atmospheric and Solar-Terrestrial Physics 72 (2010), no. 16 1195-1199. 